\title{
Book review: Land in Sicht. Ländliche Räume in Deutschland zwischen Prosperität und Peripherisierung
}

\author{
Ingo Mose \\ Institut für Biologie und Umweltwissenschaften, AG Angewandte Geographie und Umweltplanung, \\ Carl von Ossietzky Universität Oldenburg, Oldenburg, Deutschland \\ Correspondence: Ingo Mose (ingo.mose@uni-oldenburg.de) \\ Published: 17 March 2021
}

Krajewski, C.; Wiegandt, C.-C. (Hrsg.): Land in Sicht. Ländliche Räume in Deutschland zwischen Prosperität und Peripherisierung, Bundeszentrale für politische Bildung, Bonn, 410 ff., ISBN 978-3-7425-0362-6, EUR 4,50, 2020.

Land in Sicht!

Ländliche Räume haben Konjunktur. Selten hat sich binnen kurzer Zeit im deutschsprachigen Raum eine derartige Vielzahl neuer wissenschaftlicher Publikationen geographisch-raumwissenschaftlicher Provenienz, Monographien, Sammelbände und Neuauflagen älterer Publikationen, zum Thema verzeichnen lassen wie allein in den beiden zurückliegenden Jahren. Als vorerst Letzte in der Reihe haben die Geographen Christian Krajewski (Münster) und Claus-Christian Wiegandt (Bonn) Kurs auf das Thema genommen und vermelden der Leserschaft im Titel ihres von der Bundeszentrale für politische Bildung angeregten und herausgegebenen Sammelbandes unüberlesbar „Land in Sicht"! Das plakative Wortspiel greift das Motto einer vom deutschen Bundespräsidenten Frank-Walter Steinmeier durchgeführten Reihe von Besuchen auf, die dieser 2018 und 2019 verschiedenen ländlichen Regionen Deutschlands widmete. Die Land-Reisen des Staatsoberhaupts und den vorliegenden Sammelband verbindet indes mehr als ein Motto. Dem einen wie den anderen ging und geht es darum, ländliche Räume in Deutschland stärker in das Bewusstsein der Öffentlichkeit zu rücken und den dort lebenden Menschen die Aufmerksamkeit und Wertschätzung zuteilwerden zu lassen, die ihnen gebührt.

Es ist allerdings nicht so, dass ländliche Räume ein ,neues Thema“ wären. Im Gegenteil wird zumindest seitens einer Geographie ländlicher Räume seit mindestens zwei Jahrzehnten ein anhaltend intensiver Diskurs zu Fragen der ländlichen Entwicklung geführt, der ohne Zweifel deutlich we- niger konjunkturellen Schwankungen unterlag als die politische Diskussion hierzu. Eine stattliche Zahl von Workshops, Tagungen und nicht zuletzt Veröffentlichungen unterschiedlichster Art ist Ausdruck des regen Forschungsbetriebes, der sich um dieses Themenfeld entwickelt hat. Indes hat der Diskurs in den letzten Jahren offenbar eine Zuspitzung erfahren: Galten ländliche Räume schon seit jeher häufig als strukturschwach oder unterentwickelt, wurden gar als „Restgröße“ der Raumentwicklung deklariert, ist in letzter Zeit vermehrt von einem „Abgehängtsein“ vieler Regionen die Rede, das sich in handfesten infrastrukturellen, wirtschaftlichen und kulturellen Defiziten manifestiere. Gleichzeitig unterliegen ländliche Räume weiterhin einer anhaltenden Idealisierung, die mit ihnen das idyllische Bild einer vermeintlich heilen Welt assoziiert. Insbesondere im Lichte der Diskussion um die politischen Erfordernisse gleichwertiger Lebensverhältnisse in allen Teilen des Landes, die in Deutschland eine lange Tradition hat, wirken derart plakative Zuschreibungen ausgesprochen unangemessen, weil sie das Thema entweder verharmlosen oder dramatisch überspitzen und somit einer sachgerechten Diskussion im Wege stehen.

Umso willkommener sind angesichts dessen Versuche, ländliche Räume und die sie charakterisierenden Entwicklungsprozesse in differenzierter und zugleich kritischer Weise zu thematisieren und damit einer realitätsnahen Betrachtung zu verhelfen, die immer noch keine Selbstverständlichkeit ist. So auch der Anspruch des vorliegenden Sammelbandes, der hierzu einen weiteren, aktuellen Beitrag leisten will. Annähernd 40 Autor*innen, teils aus der Wissenschaft, teils aus der außeruniversitären Praxis, ausnahmslos in Deutschland tätig, haben die beiden Herausgeber um sich versammelt und, so ließe sich in Anspielung auf den Titel des Bandes formulieren, für die Aufgabe gewinnen können gemeinsam aufs Land zu gehen! Die 26 Einzelbeiträge, die im Zuge 
dieses Unterfangens verfasst wurden, sind allesamt anregend geschrieben und gut lesbar, zudem mit einer Vielzahl von Materialien, fast durchweg farbige Fotos, Karten und Tabellen, reichhaltig illustriert. Besondere Erwähnung verdient die auf dem Vorsatz des Bandes platzierte Deutschlandkarte, die zahlreiche Regionen (Landkreise, Landschaften) zeigt, die in den Beiträgen Erwähnung finden. Insbesondere eine Leserschaft, die jenseits des Wissenschaftsbetriebes Interesse an dem Thema zeigt (und sich gelegentliche geographische Orientierungshilfen wünscht), dürfte Gefallen an der graphischen Unterstützung finden.

Sechs Kapitel geben dem Band Struktur. Sie sind nach thematischen Schwerpunkten angelegt und umspannen von der Ökonomie ländlicher Räume über die Daseinsvorsorge, die Politiken der ländlichen Entwicklung und das Zusammenleben zwischen Dorf und Provinzstadt bis zum Alltag ländlicher Bevölkerungen ein weites Spektrum teils klassischer, teils moderner, zeitgenössischer Perspektiven. Während dabei teilweise durchaus Bekanntes (deshalb aber nicht weniger Wichtiges) präsentiert wird, wartet der Band zugleich mit Beiträgen von einigem Neuigkeitswert auf. So ist ein Kapitel ländlichen Räumen als Orten sozialer Innovationen gewidmet, ein anderes dem politischen Alltag in ländlichen Räumen. Zudem bietet eine Sammlung regionaler Fallbeispiele aus Ost und West interessante Einblicke im Detail. Mit Erzgebirge und Eifel werden bisher eher wenig bekannte Beispiele peripherer ländlicher Räume vorgestellt, über die die Autor*innen einiges zu sagen wissen, während das Emsland zum wiederholten Mal als Beispiel einer erfolgreichen Entwicklung bemüht wird, obwohl darüber kaum Neues mehr zu Tage gefördert werden kann. Willkommene Abwechslungen vom dominanten Aufsatzformat stellen zwei Interviews dar, von denen sich durchaus noch weitere wünschen ließen. Erklärtermaßen ist der Fokus des Bandes auf Deutschland gerichtet, gleichwohl viele Beiträge so angelegt sind, dass sie auch eine Leserschaft in der Schweiz und Österreich interessieren könnten. Auch wenn sich für einen Sammelband wie diesen kaum der Anspruch auf Vollständigkeit erheben lässt, dürfen einige Desiderate nicht unerwähnt bleiben. So wird in keinem Beitrag die nachschulische Bildung thematisiert, ebenso wenig die Bedeutung von Universitäten, Hochschulen und sonstigen Forschungseinrichtungen für die ländliche Entwicklung. Eine auffällige Leerstelle bildet zudem das weite Themenfeld des Umwelt-, Klima- und Naturschutzes, auf das der Band nur wenige punktuelle Hinweise liefert. Angesichts der wachsenden Bedeutung, die den damit verbundenen Herausforderungen beigemessen wird, wäre hierzu durchaus ein eigenes Kapitel zu erwarten gewesen.
Qualität und Wert der vorliegenden Publikation tut dies jedoch keinen merklichen Abbruch: Den beiden Herausgebern gebührt vielmehr Dank dafür, die zweifellos mühevolle Zusammenstellung eines Sammelbandes übernommen zu haben, der den Kanon der aktuellen Veröffentlichungen um eine wichtige Facette erweitert. Mit ihrem Buch präsentiert sich ein gleichermaßen aktueller wie differenzierter Überblick zu (fast) allen relevanten Themen, die gegenwärtig die Diskussion um die Herausforderungen und Zukunftsperspektiven ländlicher Räume in Deutschland bestimmen. Einmal mehr wird damit deutlich, dass es eine homogene Kategorie des ländlichen Raumes in Deutschland nicht gibt, sondern sinnvoll nur von ländlichen Räumen im Plural gesprochen werden kann, deren Entwicklung sich in einem Spannungsfeld zwischen Prosperität und Peripherisierung (so der Untertitel des Buches) vollzieht. Ein solches Werk hat bisher gefehlt; es erscheint zur rechten Zeit und ist höchst willkommen - nicht nur der Vielzahl an Informationen wegen, die es transportiert, sondern auch der Anregungen zur kritischen Auseinandersetzung, die von ihm ausgehen. Fast ungläubig schaut man auf den Preis von sage und schreibe EUR 4,50, zu dem die Bundeszentrale für politische Bildung das Buch vertreibt. Es sei allen Interessierten auf das Wärmste empfohlen! 